\title{
Asymptomatic COVID-19 among individuals living with diabetes: Rapid scoping review
}

\author{
Nwose EU1,2* and Bwititi PT ${ }^{3}$ \\ ${ }^{1}$ School of Community Health, Charles Sturt University, Orange, NSW Australia \\ ${ }^{2}$ Department of Public \& Community Health, Novena University, Ogume, Nigeria \\ ${ }^{3}$ School of Biomedical Sciences, Charles Sturt University, Wagga Wagga, NSW Australia
}

\begin{abstract}
Background and Aim: There is concern over the novel coronavirus (COVID-19) in individuals with diabetes mellitus. In the ongoing discourse, interest arises "if medicines we already have are good enough to buy patients more time" and whether diabetes drugs are friends or foe. This study focused on whether oral hypoglycaemic drugs constitute complementary medicine for COVID-19 in those living with diabetes.
\end{abstract}

Method: Rapid scoping review was done and articles on PubMed and research News related to COVID19 were screened. Focus was outcome of asymptomatic COVID-19 rehabilitative management in people living with diabetes.

Results: From over 14,600 articles, 3 papers were selected and critically appraised. Up to 30\% of COVID-19 cases may have diabetes as pre-existing and most will be symptomatic of which about a quarter may aggravate. This prevalence of aggravation is higher than in the subpopulation with only respiratory disease. Among the asymptomatic subpopulation, rehabilitation seems absolutely and most successful in preventing progression to symptomatic status when compared to other COVID-19 subpopulations.

Conclusion: The results signify that some of the medicines already available are good enough to buy COVID-19 patients time. However, majority of COVID-19 cases with pre-existing diabetes could be symptomatic and likely to aggravate. While routine clinical management may be doubling up as complementary medicine for COVID-19, effect may be observable in those without symptoms. Concern on glucocorticoid use is warranted, especially in diabetes clients who are immunocompromised that may worsen with the therapy.

\section{Highlights}

- Up to 30\% COVID19 cases have pre-existing DM and most are symptomatic. Glucocorticoid is common anti-inflammatory drug used.

- In symptomatic COVID19 cases: aggravation has been recorded among those with diabetes than in respiratory disease.

- In asymptomatic COVID19 cases: best management outcome has been recorded in diabetes subgroup compared to other conditions.

- Antioxidant and immunomodulatory properties of hypoglycaemics may be helping while glucocorticoids have worsening effects.

\section{Introduction}

There is concern over the novel coronavirus (COVID-19) among individuals with diabetes mellitus. In the treatment roadmap of COVID-19; there are options including vaccine, anti-inflammatories, management of symptoms and complementary medicine. In the ongoing discourse, interest arises "if medicines we have are good enough to buy patients more time" [1].

Specifically for those living with diabetes, there is related interest whether diabetes drugs are friends or foe [2]. Therefore, this study focuses on whether oral hypoglycaemic drugs constitute complementary medicine for COVID-19 in diabetes. This objective is in line with suggestion to investigate outcomes data with regards to the effects of hypoglycaemic drugs [3].

\section{Method}

This was a rapid scoping review that followed two processes. The search terms on PubMed were in add-on sequence of COVID-19, diabetes, asymptomatic and treatment with no time bound applied. Additional process was review of research News updates - with vigilance for reports involving asymptomatic COVID-19 comorbidity with diabetes and follow-up evaluations. The main interest was to compare outcome of asymptomatic COVID-19 rehabilitative management in people living with diabetes.

\section{Results}

Over 14600 articles on COVID-19 have been indexed in the searched database, and 3\% involved diabetes. However, only 7 papers appeared to include asymptomatic considerations (Table 1), of which 2 were critically reviewed $[4,5]$; and one delineated those with diabetes who were COVID-19 asymptomatic.

*Correspondence to: Uba Nwose, School of Community Health, Charles Sturt University, NSW 2800 Australia, Tel: +6126365 7282; E-mail: enwose@csu. edu.au

Key words: COVID19 pandemic, complementary medicine, diabetes mellitus, hypoglycaemic drugs, management outcomes

Received: December 30, 2020; Accepted: January 25, 2021; Published: January 28,2021 
Table 1. Sequence and outcome of literature search

\begin{tabular}{|l|l|l|}
\hline Steps & Search term & No. of articles \\
\hline 1 & COVID19 & 14602 \\
\hline 2 & COVID19//diabetes & 427 \\
\hline 3 & COVID19//diabetes//asymptomatic & 7 \\
\hline 4 & COVID19//diabetes//asymptomatic//reatment & 2 \\
\hline
\end{tabular}

In the research News updates, one was retrospective evaluation and categorized the diabetes subpopulation into asymptomatic versus non-asymptomatic [6]. Therefore, 3 papers were critical appraised and summary results show that:

Approximately $30 \%$ may comprise coexisting DM and a significant minority could be asymptomatic. Besides antimicrobial agents, glucocorticoid is the common anti-inflammatory drug for the COVID-19 [5].

Disease aggravation could be about a quarter (26\%) of people living with diabetes. This level of aggravation is high when compared to the subpopulation of those with only pre-existing respiratory disease [4].

Comparing management outcome in those without symptoms (i.e. asymptomatic COVID-19 with diabetes or other pre-existing conditions), rehabilitation may be most successful among the diabetes group (Figure 1) [6].

Considering effect of the management on haematological indices for immune response, further results show average $23 \%$ of those who progress have low lymphocyte, platelet and white blood cell counts. Among the subgroup who did not progress, averaged $14 \%$ have low counts of the haematological indices (Figure 2).

\section{Discussion}

This brief review surmises common phenomenon in research whereby perspectives of observations differ according to objectives and protocols. Here, three different perspectives present a holistic view. First, while COVID-19 patients may have pre-existing diabetes, glucocorticoid may also be used in the treatment. This is important because of the hyperglycaemic and immunosuppression side-effects of glucocorticoids. Arguably, glucocorticoids should be contraindicated in immunocompromised COVID-19 patients $[7,8]$. This contraindication may be relevant in diabetes and geriatrics [3,9], especially considering the glucocorticoids side-effects of worsening both hyperglycaemia and immunosuppression.

Secondly, disease aggravation appears to be in a smaller fraction of the diabetes subpopulation. While this is subject to validation, it is pertinent to note that such aggravation is less in the subpopulation with pre-existing respiratory disease. Third and more striking is where the COVID-19 asymptomatic cases were assessed that none of those with diabetes progressed to symptomatic status of the respiratory infection (Figure 1).

We previously reported on a family case of metabolic syndrome [10], and one family member died of disseminated intravascular coagulation (DIC) complication in 2018. In the ongoing COVID-19 pandemic, a recent report has indicated DIC as the complication underlying the deaths [11]. Therefore, this has drawn our interest on relevant interaction in COVID-19-diabetes comorbidity. It suffices to note that the interaction can be in the pathophysiological processes as well as by drug-health interaction.

In terms of pathophysiology, oxidative stress is involved in diabetes and athero-thrombogenic process $[12,13]$. Hence there is application of antioxidant micronutrients in diabetes management
[14]. Mitochondria damage is involved in several diseases and includes mitochondrial ROS production in platelets, which constitutes part of risk of DIC [15]. Thus, a pre-existing disease such as diabetes can exacerbate athero-thrombogenesis en-route formation of DIC. In this report, results indicate that coronavirus disease may be aggravated more in diabetics than in those without diabetes. What this report contribute to the ongoing discourse is a reminder albeit what is already known that oxidative stress damage leading to DIC is probably exacerbated in COVID-19 comorbidity with diabetes.

In terms of treatment interactions: Antioxidants have immunomodulatory effect [16]; and have been applicable in diabetes management. Antioxidants remove free radicals to reduce DIC formation process [17]. For instance, metformin has been discussed and evaluated for antioxidant and immunomodulatory functions. In terms of the COVID-19 pandemic, it is necessary to re-articulate how diabetes drugs with antioxidant cum immunomodulatory properties can be appropriated in management of COVID-19 patients with diabetes.

The relevance of this discourse is important with regard to "if medicines we already have are good enough to buy patients more time" [1]; and whether diabetes drugs are friends or foe [2]. In regard to the study report reviewed and graphically re-presented (Figure 1), the non-progression of asymptomatic COVID-19 infected individuals living with diabetes can only be attributed to their hypoglycaemia management. This notion is supported by the potential of antioxidant and immunomodulatory effects of hypoglycaemic drugs including metformin [18,19].

This review makes a point that symptomatic people with COVID-19 who have diabetes are more likely to progress to severe stages due to complex pathophysiological processes including but

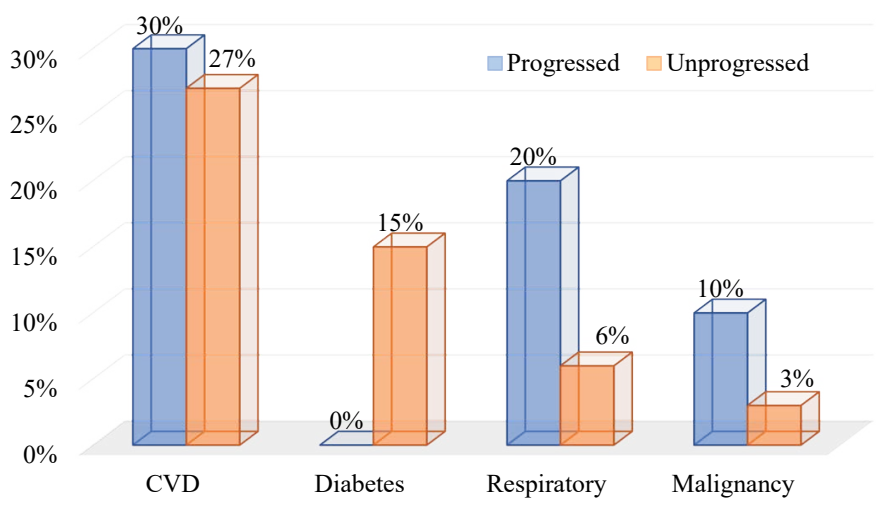

Figure 1. Clinical characteristics of 43 asymptomatic COVID19 patients on follow-up

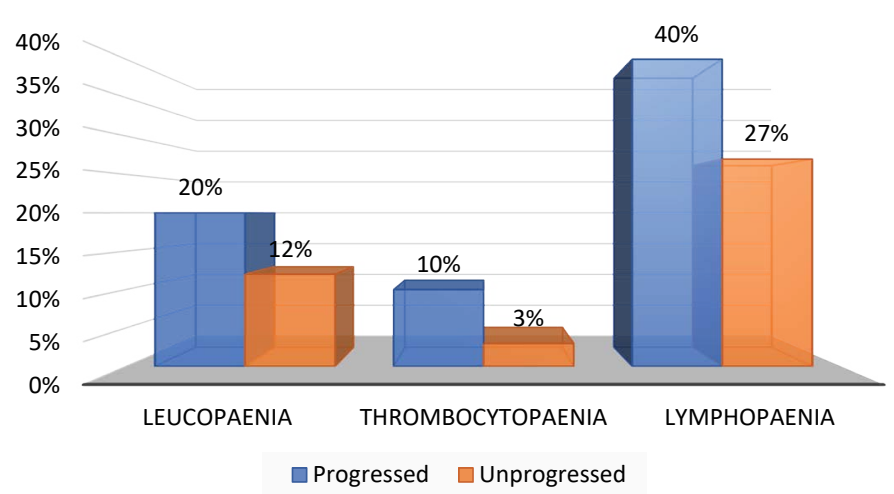

Figure 2. Effects of management on haematological indices of immune response 
not limited to aggravated immunosuppression possibly arising from side-effects of anti-inflammatory drugs that may worsen both glycaemic control and immune status. On the other hand, asymptomatic people with COVID-19 who have diabetes are less likely to aggravate. Possible reason is that in the absence of polypharmacy, the role of antihyperglycemic therapy may include antioxidant cum immunomodulatory properties.

From the clinical laboratory perspective, there is no gain saying the fact that routing full blood count constitute a valid tool for assessment. In the case reviewed, reported data show comparison of the subgroup who progressed relative to the non-progressed - re: abnormally low counts are more in the progressed group (Figure 2). It may also benefit to note that the risk of DIC can be assessed by whole blood viscosity using routine haematocrit and serum protein tests [20], which are available in most pathology services.

To conclude, this rapid review submits that some of the medicines already available are good and effectively 'buying patients more time'. The treatment outcomes of diabetes drugs may be more evident in the asymptomatic COVID19 patients. However, the majority of cases with pre-existing diabetes may be symptomatic and likely to aggravate.

\section{References}

1. https://www.wired.com/story/how-un-miracle-drugs-could-help-tame-the-pandemic/

2. Ursini F, Ciaffi J, Landini MP, Meliconi R (2020) COVID-19 and diabetes: Is metformin a friend or foe? Diabetes Res Clin Pract 164: 108167. [Crossref]

3. Desai R, Singh S, Parekh T, Sachdeva S, Sachdeva R, et al. (2020) COVID-19 and diabetes mellitus: A need for prudence in elderly patients from a pooled analysis. Diabetes Metab Syndr 14: 683-685. [Crossref]

4. Wang X, Fang J, Zhu Y, Chen L, Ding F, et al. (2020) Clinical characteristics of noncritically ill patients with novel coronavirus infection (COVID-19) in a Fangcang Hospital. Clin Microbiol Infect 26: 1063-1068. [Crossref]

5. Xiong F, Tang H, Liu L, Tu C, Tian J, et al. (2020) Clinical characteristics of and medical interventions for COVID-19 in hemodialysis patients in Wuhan, China. J Am Soc Nephrol 31: 1387-1397. [Crossref]

6. Tabata S, Imai K, Kawano S, et al. The clinical characteristics of COVID-19: a retrospective analysis of 104 patients from the outbreak on board the Diamond Princess cruise ship in Japan. medRxiv.
7. Zha L, Li S, Pan L, Tefsen B, Li Y, et al. (2020) Corticosteroid treatment of patients with coronavirus disease 2019 (COVID-19). Med J Aust 212: 416-420. [Crossref]

8. Russell B, Moss C, Rigg A, Hemelrijck MV (2020) COVID-19 and treatment with NSAIDs and corticosteroids: should we be limiting their use in the clinical setting? Ecancermedicalscience 14: 1023-1023. [Crossref]

9. Marhl M, Grubelnik V, Magdič M, Markovič R (2020) Diabetes and metabolic syndrome as risk factors for COVID-19. Diabetes Metab Syndr 14: 671-677. [Crossref]

10. Anyasodor AE, Nwose EU, Richards RS, Bwititi PT, Mudiaga LI, et al. (2017) Prediabetes and cardiovascular complications screening in Nigeria: A family case presentation. Diabetes Metab Syndr 11: 273-275. [Crossref]

11. Watanabe T, Imamura T, Nakagaki K (1979) Disseminated intravascular coagulation in autopsy cases its incidence and clinicopathologic significance. Pathology - Research and Practice 165: 311-322.

12. Pawlak K, Naumnik B, Brzósko S (2004) Oxidative Stress - a Link between Endothelial Injury, Coagulation Activation, and Atherosclerosis in Haemodialysis Patients. American Journal of Nephrology 24: 154-161.

13. Nwose EU, Jelinek HF, Richards RS, Kerr PG (2007) Erythrocyte oxidative stress in clinical management of diabetes and its cardiovascular complication. Br J Biomed Sci 64: 35-43. [Crossref]

14. Sfar S, Jawed A, Braham H, Amor S, Laporte F, et al. (2009) Zinc, copper and antioxidant enzyme activities in healthy elderly Tunisian subjects. Exp Gerontol 44: 812-817. [Crossref]

15. Melchinger H, Jain K, Tyagi T, John Hwa (2019) Role of platelet mitochondria: Life in a nucleus-free zone. Front Cardiovasc Med 6: 153. [Crossref]

16. Li X-L, Zhou A-G, Zhang L, Chen W (2011) Antioxidant status and immune activity of glycyrrhizin in allergic rhinitis mice. Int J Mol Sci 12: 905-916. [Crossref]

17. Pawlak K, Borawski J, Naumnik B (2003) Relationship between oxidative stress and extrinsic coagulation pathway in haemodialyzed patients. Thrombosis Research 109 $247-251$

18. Chukwunonso Obi B, Chinwuba Okoye T, Okpashi VE, Igwe CN, Alumanah EO, et al (2016) Comparative study of the antioxidant effects of metformin, glibenclamide, and repaglinide in alloxan-induced diabetic rats. J Diabetes Res 2016: 1635361. [Crossref]

19. Choi SW, Ho CK (2018) Antioxidant properties of drugs used in Type 2 diabetes management: could they contribute to, confound or conceal effects of antioxidant therapy? Redox Rep 23: 1-24. [Crossref]

20. Nwose EU (2010) Whole blood viscosity assessment issues II: prevalence in endothelial dysfunction and hypercoagulation. North Am J Med Sci 2: 252-257. [Crossref]

Copyright: (C2021 Nwose EU. This is an open-access article distributed under the terms of the Creative Commons Attribution License, which permits unrestricted use, distribution, and reproduction in any medium, provided the original author and source are credited. 